\title{
Oscillation for a Class of Second-Order Emden-Fowler Delay Dynamic Equations on Time Scales
}

\author{
Shurong Sun, ${ }^{1,2}$ Zhenlai Han, ${ }^{1,3}$ Ping Zhao, ${ }^{4}$ and Chao Zhang ${ }^{1}$ \\ ${ }^{1}$ School of Science, University of Jinan, Jinan, Shandong 250022, China \\ ${ }^{2}$ Department of Mathematics and Statistics, Missouri University of Science and Technology, Rolla, \\ MO 65409-0020, USA \\ ${ }^{3}$ School of Control Science and Engineering, Shandong University, Jinan, Shandong 250061, China \\ ${ }^{4}$ School of Control Science and Engineering, University of Jinan, Jinan, Shandong 250022, China
}

Correspondence should be addressed to Shurong Sun, sshrong@163.com

Received 13 December 2009; Accepted 4 March 2010

Academic Editor: Leonid Berezansky

Copyright (C) 2010 Shurong Sun et al. This is an open access article distributed under the Creative Commons Attribution License, which permits unrestricted use, distribution, and reproduction in any medium, provided the original work is properly cited.

By means of Riccati transformation technique, we establish some new oscillation criteria for the second-order Emden-Fowler delay dynamic equations $\left(r x^{\Delta}\right)^{\Delta}(t)+p(t) x^{\gamma}(\tau(t))=0$ on a time scale $\mathbb{T}$; here $\gamma$ is a quotient of odd positive integers with $r$ and $p$ as real-valued positive rd-continuous functions defined on $\mathbb{T}$. Our results in this paper not only extend the results given in Agarwal et al. (2005), Akin-Bohner et al. (2007) and Han et al. (2007) but also unify the results about oscillation of the second-order Emden-Fowler delay differential equation and the second-order Emden-Fowler delay difference equation.

\section{Introduction}

The theory of time scales, which has recently received a lot of attention, was introduced by Hilger in his Ph.D. Thesis in 1988 in order to unify continuous and discrete analysis (see Hilger [1]). Several authors have expounded on various aspects of this new theory; see the survey paper by Agarwal et al. [2] and references cited therein. A book on the subject of time scales, by Bohner and Peterson [3], summarizes and organizes much of the time scale calculus; we refer also to the last book by Bohner and Peterson [4] for advances in dynamic equations on time scales. For the notions used below, we refer to the next section that provides some basic facts on time scales extracted from Bohner and Peterson [3].

A time scale $\mathbb{T}$ is an arbitrary closed subset of the reals, and the cases when this time scale is equal to the reals or to the integers represent the classical theories of differential and 
of difference equations. Not only does the new theory of the so-called dynamic equations unify the theories of differential equations and difference equations, but also it extends these classical cases to cases in between, for example, to the so-called $q$-difference equations when $\mathbb{T}=q^{\mathbb{N}_{0}}$ which has important applications in quantum theory and can be applied on different types of time scales like $\mathbb{T}=h \mathbb{Z}, \mathbb{T}=\mathbb{N}_{0}^{2}$, and $\mathbb{T}=\mathbb{T}_{n}$ the space of the harmonic numbers.

Many other interesting time scales exist, and they give rise to plenty of applications, among them the study of population dynamic models which are discrete in season (and may follow a difference scheme with variable step-size or often modeled by continuous dynamic systems), die out, say in winter, while their eggs are incubating or dormant, and then in season again, hatching gives rise to a nonoverlapping population (see Bohner and Peterson $[3])$.

In recent years, there has been much research activity concerning the oscillation and nonoscillation of solutions of various equations on time scales, and we refer the reader to Akin-Bohner and Hoffacker [5, 6], Akin-Bohner et al. [7], Bohner and Saker [8], Erbe [9], Erbe et al. [10], Li et al. [11], and Saker [12, 13]. However, there are few results dealing with the oscillation of the solutions of delay dynamic equations on time scales [14-29].

Following this trend, in this paper, we consider a second-order nonlinear delay differential equation

$$
\left(r x^{\Delta}\right)^{\Delta}(t)+p(t) x^{r}(\tau(t))=0 \quad \text { for } t \in \mathbb{T}
$$

For oscillation of the second-order delay dynamic equations, Agarwal et al. [14] considered the second-order delay dynamic equations on time scales

$$
x^{\Delta \Delta}(t)+p(t) x(\tau(t))=0, \quad t \in \mathbb{T},
$$

and established some sufficient conditions for oscillation of (1.2).

Zhang and Shanliang [29] studied the second-order nonlinear delay dynamic equations on time scales

$$
x^{\Delta \Delta}(t)+p(t) f(x(t-\tau))=0, \quad t \in \mathbb{T},
$$

and the second-order nonlinear dynamic equations on time scales

$$
x^{\Delta \Delta}(t)+p(t) f(x(\sigma(t)))=0, \quad t \in \mathbb{T},
$$

where $\tau \in \mathbb{R}$ and $t-\tau \in \mathbb{T}, f: \mathbb{R} \rightarrow \mathbb{R}$ is continuous and nondecreasing $\left(f^{\prime}(u)>k>0\right)$, and $u f(u)>0$ for $u \neq 0$, and established the equivalence of the oscillation of (1.3) and (1.4). However, the results established in [29] are valid only when the graininess function $\mu(t)$ is bounded which is a restrictive condition. Also the restriction $f^{\prime}(u)>k>0$ is required.

Şahiner [23] considered the second-order nonlinear delay dynamic equations on time scales

$$
x^{\Delta \Delta}(t)+p(t) f(x(\tau(t)))=0, \quad t \in \mathbb{T},
$$


where $f: \mathbb{R} \rightarrow \mathbb{R}$ is continuous, $u f(u)>0$ for $u \neq 0$ and $|f(u)| \geq L|u|, \tau: \mathbb{T} \rightarrow \mathbb{T}, \tau(t) \leq t$, and $\lim _{t \rightarrow \infty} \tau(t)=\infty$ and obtained some sufficient conditions for oscillation of (1.5).

Han et al. [17] investigated the second-order Emden-Fowler delay dynamic equations on time scales

$$
x^{\Delta \Delta}(t)+p(t) x^{\gamma}(\tau(t))=0, \quad t \in \mathbb{T},
$$

established some sufficient conditions for oscillation of (1.6), and extended the results given in [14].

Erbe et al. [28] considered the general nonlinear delay dynamic equations on time scales

$$
\left(p(t) x^{\Delta}(t)\right)^{\Delta}+q(t) f(x(\tau(t)))=0, \quad t \in \mathbb{T}
$$

where $p$ and $q$ are positive, real-valued rd-continuous functions defined on $\mathbb{T}, \tau: \mathbb{T} \rightarrow \mathbb{T}$ is rd-continuous, $\tau(t) \leq t$ and $\tau(t) \rightarrow \infty$ as $t \rightarrow \infty$, and $f \in C(\mathbb{R}, \mathbb{R})$ satisfies for some positive constant $L,|f(x)| \geq L|x|, x f(x)>0$ for all nonzero $x$, and extended the generalized Riccati transformation techniques in the time scales setting to obtain some new oscillation criteria which improve the results given by Şahiner [23] and Zhang and Shanliang [29].

Clearly, (1.2), (1.3), (1.5), (1.6), and (1.7) are different from (1.1). To develop the qualitative theory of delay dynamic equations on time scales, in this paper, we consider the second-order nonlinear delay dynamic equation on time scales (1.1).

As we are interested in oscillatory behavior, we assume throughout this paper that the given time scale $\mathbb{T}$ is unbounded above, that is, it is a time scale interval of the form $[a, \infty)$ with $a \in \mathbb{T}$.

We assume that $\gamma$ is a quotient of odd positive integer, $r$ and $p$ are positive, real-valued rd-continuous functions defined on and $\mathbb{T}, \tau: \mathbb{T} \rightarrow \mathbb{T}$ is a rd-continuous function such that $\tau(t) \leq t$ and $\tau(t) \rightarrow \infty(t \rightarrow \infty)$.

We shall also consider the two cases

$$
\begin{aligned}
& \int_{a}^{\infty} \frac{1}{r(t)} \Delta t=\infty, \\
& \int_{a}^{\infty} \frac{1}{r(t)} \Delta t<\infty .
\end{aligned}
$$

By a solution of (1.1), we mean a nontrivial real-valued function $x$ satisfying (1.1) for $t \geq t_{x} \geq a$. A solution $x$ of (1.1) is called oscillatory if it is neither eventually positive nor eventually negative; otherwise, it is called nonoscillatory. Equation (1.1) is called oscillatory if all solutions are oscillatory. Our attention is restricted to those solutions $x$ of (1.1) which exist on some half line $\left[t_{x}, \infty\right)$ with $\sup \left\{|x(t)|: t \geq t_{0}\right\}>0$ for any $t_{0} \geq t_{x}$.

We note that if $\mathbb{T}=\mathbb{R}$, then $\sigma(t)=t, \mu(t)=0, x^{\Delta}(t)=x^{\prime}(t)$, and (1.1) becomes the second-order Emden-Fowler delay differential equation

$$
\left(r x^{\prime}\right)^{\prime}(t)+p(t) x^{\gamma}(\tau(t))=0 \quad \text { for } t \in \mathbb{R} .
$$


If $\mathbb{T}=\mathbb{Z}$, then $\sigma(t)=t+1, \mu(t)=1, x^{\Delta}(t)=\Delta x(t)=x(t+1)-x(t)$, and (1.1) becomes the second-order Emden-Fowler delay difference equation

$$
\Delta(r(t) \Delta x(t))+p(t) x^{\gamma}(\tau(t))=0 \quad \text { for } t \in \mathbb{Z}
$$

In the case of $\gamma>1,(1.1)$ is the prototype of a wide class of nonlinear dynamic equations called Emden-Fowler superlinear dynamic equations, and if $0<\gamma<1$, then (1.1) is the prototype of dynamic equations called Emden-Fowler sublinear dynamic equations. It is interesting to study (1.1) because the continuous version, that is, (1.10), has several physical applications—see, for example, [1] —and when $t$ is a discrete variable as in (1.11), (1.1) also has important applications.

Numerous oscillation and nonoscillation criteria have been established for equations as (1.10) and (1.11); see, for example, [1,30-36] and references therein.

In this paper, we intend to use the Riccati transformation technique for obtaining several oscillation criteria for (1.1). Our results in this paper not only extend the results given in Agarwal et al. [14] and Han et al. [17] but also unify the oscillation of the second-order Emden-Fowler delay differential equation and the second-order Emden-Fowler delay difference equation. Applications to equations to which previously known criteria for oscillation are not applicable are given.

This paper is organized as follows: in Section 2, we present the basic definitions and the theory of calculus on time scales. In Section 3, we apply a simple consequence of Kellers chain rule, devoted to the proof of the sufficient conditions for oscillation of all solutions of (1.1). In Section 4, some applications and examples are considered to illustrate the main results.

\section{Some Preliminaries}

A time scale $\mathbb{T}$ is an arbitrary nonempty closed subset of the real numbers $\mathbb{R}$. Since we are interested in oscillatory behavior, we suppose that the time scale under consideration is not bounded above, that is, it is a time scale interval of the form $[a, \infty) \subset \mathbb{T}$. On any time scale, we define the forward and backward jump operators by

$$
\sigma(t):=\inf \{s \in \mathbb{T} \mid s>t\}, \quad \rho(t):=\sup \{s \in \mathbb{T} \mid s<t\} .
$$

A point $t \in \mathbb{T}$ is said to be left-dense if $\rho(t)=t$, right-dense if $\sigma(t)=t$, left-scattered if $\rho(t)<t$, and right-scattered if $\sigma(t)>t$. The graininess $\mu$ of the time scale is defined by $\mu(t):=\sigma(t)-t$.

For a function $f: \mathbb{T} \rightarrow \mathbb{R}$ (the range $\mathbb{R}$ of $f$ may actually be replaced by any Banach space), the (delta) derivative is defined by

$$
f^{\Delta}(t)=\frac{f(\sigma(t))-f(t)}{\sigma(t)-t}
$$


if $f$ is continuous at $t$ and $t$ is right-scattered. If $t$ is not right-scattered, then the derivative is defined by

$$
f^{\Delta}(t)=\lim _{s \rightarrow t^{+}} \frac{f(\sigma(t))-f(s)}{t-s}=\lim _{s \rightarrow t^{+}} \frac{f(t)-f(s)}{t-s}
$$

provided this limit exists.

A function $f: \mathbb{T} \rightarrow \mathbb{R}$ is said to be rd-continuous if it is continuous at each right-dense point and if there exists a finite left limit in all left-dense points. The set of rd-continuous functions $f: \mathbb{T} \rightarrow \mathbb{R}$ is denoted by $C_{\mathrm{rd}}(\mathbb{T}, \mathbb{R})$.

$f$ is said to be differentiable if its derivative exists. The set of functions $f: \mathbb{T} \rightarrow \mathbb{R}$ that are differentiable and whose derivative is rd-continuous function is denoted by $C_{\mathrm{rd}}^{1}(\mathbb{T}, \mathbb{R})$.

The derivative and the shift operator $\sigma$ are related by the formula

$$
f^{\sigma}=f+\mu f^{\Delta}, \quad \text { where } f^{\sigma}:=f \circ \sigma .
$$

Let $f$ be a real-valued function defined on an interval $[a, b]$. We say that $f$ is increasing, decreasing, nondecreasing, and nonincreasing on $[a, b]$ if $t_{1}, t_{2} \in[a, b]$ and $t_{2}>t_{1}$ imply $f\left(t_{2}\right)>f\left(t_{1}\right), f\left(t_{2}\right)<f\left(t_{1}\right), f\left(t_{2}\right) \geq f\left(t_{1}\right)$, and $f\left(t_{2}\right) \leq f\left(t_{1}\right)$, respectively. Let $f$ be a differentiable function on $[a, b]$. Then $f$ is increasing, decreasing, nondecreasing, and nonincreasing on $[a, b]$ if $f^{\Delta}(t)>0, f^{\Delta}(t)<0, f^{\Delta}(t) \geq 0$, and $f^{\Delta}(t) \leq 0$ for all $t \in[a, b)$, respectively.

We will make use of the following product and quotient rules for the derivative of the product $f g$ and the quotient $f / g$ of two differentiable functions $f$ and $g$

$$
\begin{gathered}
(f g)^{\Delta}(t)=f^{\Delta}(t) g(t)+f(\sigma(t)) g^{\Delta}(t)=f(t) g^{\Delta}(t)+f^{\Delta}(t) g(\sigma(t)), \\
\left(\frac{f}{g}\right)^{\Delta}(t)=\frac{f^{\Delta}(t) g(t)-f(t) g^{\Delta}(t)}{g(t) g(\sigma(t))} .
\end{gathered}
$$

For $a, b \in \mathbb{T}$ and a differentiable function $f$, the Cauchy integral of $f^{\Delta}$ is defined by

$$
\int_{a}^{b} f^{\Delta}(t) \Delta t=f(b)-f(a)
$$

The integration by parts formula reads

$$
\int_{a}^{b} f^{\Delta}(t) g(t) \Delta t=f(b) g(b)-f(a) g(a)-\int_{a}^{b} f^{\sigma}(t) g^{\Delta}(t) \Delta t
$$

and infinite integrals are defined as

$$
\int_{a}^{\infty} f(s) \Delta s=\lim _{t \rightarrow \infty} \int_{a}^{t} f(s) \Delta s
$$


In case $\mathbb{T}=\mathbb{R}$, we have

$$
\sigma(t)=\rho(t)=t, \quad \mu(t) \equiv 0, \quad f^{\Delta}=f^{\prime}, \quad \int_{a}^{b} f(t) \Delta t=\int_{a}^{b} f(t) d t
$$

And in case $\mathbb{T}=\mathbb{Z}$, we have

$$
\sigma(t)=t+1, \quad \rho(t)=t-1, \quad \mu(t) \equiv 1, \quad f^{\Delta}=\Delta f, \quad \int_{a}^{b} f(t) \Delta t=\sum_{t=a}^{b-1} f(t) .
$$

\section{Main Results}

In this section, we give some new oscillation criteria for (1.1). Since we are interested in oscillatory behavior, we will suppose that the time scale $\mathbb{T}$ under consideration is not bounded above, that is, it is a time scale interval of the form $[a, \infty)$. In order to prove our main results, we will use the formula

$$
\left((x(t))^{\gamma}\right)^{\Delta}=\gamma \int_{0}^{1}\left[h x^{\sigma}(t)+(1-h) x(t)\right]^{\gamma-1} x^{\Delta}(t) d h
$$

where $x$ is delta differentiable and eventually positive or eventually negative, which is a simple consequence of Keller's chain rule (see Bohner and Peterson [3, Theorem 1.90]). Also, we need the following auxiliary result.

Lemma 3.1 (Şahiner [23, Lemma 1]). Suppose that the following conditions hold:

$\left(\mathrm{H}_{1}\right) u \in C_{\mathrm{rd}}^{2}(I, \mathbb{R})$, where $I=\left[t_{*}, \infty\right) \subset \mathbb{T}$ for some $t_{*}>0$

$\left(\mathrm{H}_{2}\right) u(t)>0, u^{\Delta}(t)>0$ and $u^{\Delta \Delta}(t) \leq 0$ for $t \geq t_{*}$.

Then, for each $k \in(0,1)$, there exists a constant $t_{k} \in \mathbb{T}, t_{k} \geq t_{*}$, such that

$$
u(\sigma(t)) \leq \frac{\sigma(t)}{k \tau(t)} u(\tau(t)) \quad \text { for } t \geq t_{k}
$$

Lemma 3.2. Assume that (1.8) holds. Furthermore, assume that $r \in C_{\mathrm{rd}}^{1}([a, \infty), \mathbb{R}), r^{\Delta}(t) \geq 0$, and $x(t)$ is an eventually positive solution of (1.1). Then, there exists $a t_{1} \geq a$ such that

$$
x^{\Delta}(t)>0, \quad x^{\Delta \Delta}(t)<0, \quad\left(r(t) x^{\Delta}(t)\right)^{\Delta}<0 \quad \text { for } t \geq t_{1}
$$

Proof. Suppose $x(t)$ is an eventually positive solution of (1.1) such that $x(t)>0$ and $x(\tau(t))>$ 0 for all $t \geq t_{0}>a$. In view of (1.1), we have

$$
\left(r(t) x^{\Delta}(t)\right)^{\Delta}=-p(t)(x(\tau(t)))^{\gamma}<0 \quad \text { for } t \geq t_{0},
$$


and so $r(t) x^{\Delta}(t)$ is an eventually decreasing function. We first show that $r(t) x^{\Delta}(t)$ is eventually positive. Indeed, the decreasing function $r(t) x^{\Delta}(t)$ is either eventually positive or eventually negative. Suppose that there exists an integer $t_{1} \geq t_{0}$ such that $r\left(t_{1}\right) x^{\Delta}\left(t_{1}\right)=c<0$, then from (3.4) we have $r(t) x^{\Delta}(t) \leq r\left(t_{1}\right) x^{\Delta}\left(t_{1}\right)=c$ for $t \geq t_{1}$, hence

$$
x^{\Delta}(t) \leq \frac{c}{r(t)}
$$

which implies by (1.8) that

$$
x(t) \leq x\left(t_{1}\right)+c \int_{t_{1}}^{t} \frac{1}{r(s)} \Delta s \longrightarrow-\infty, \quad \text { as } t \longrightarrow \infty,
$$

and this contradicts the fact that $x(t)>0$ for all $t \geq t_{0}$. Hence $r(t) x^{\Delta}(t)$ is eventually positive. So $x^{\Delta}(t)$ is eventually positive. Then $x(t)$ is eventually increasing.

By (2.5), we get

$$
\left(r(t) x^{\Delta}(t)\right)^{\Delta}=r^{\Delta}(t) x^{\Delta}(t)+r(\sigma(t)) x^{\Delta \Delta}(t)
$$

From (3.4), (3.7), and $r^{\Delta}(t) \geq 0$, we can easily verify that $x^{\Delta \Delta}(t)$ is eventually negative. Therefore, we see that there is some $t_{1} \geq t_{0}$ such that (3.3) holds. The proof is complete.

Lemma 3.3. Let $z$ and $x$ be differentiable on time scale $\mathbb{T}$ with $x(t) \neq 0$ for all $t \in \mathbb{T}$. Then we have

$$
\left(x^{\gamma}\right)^{\Delta}\left(\frac{z^{2}}{x^{\gamma}}\right)^{\Delta}=\left(z^{\Delta}\right)^{2}-\left(x x^{\sigma}\right)^{\gamma}\left(\left(\frac{z}{x^{\gamma}}\right)^{\Delta}\right)^{2} .
$$

The proof is similar to that of Akin-Bohner et al. [7, Lemma 3.1].

Theorem 3.4. Assume that (1.8) holds, $r \in C_{\mathrm{rd}}^{1}([a, \infty), \mathbb{R})$, and $r^{\Delta}(t) \geq 0, r>1$. Define

$$
R(t)=\int_{a}^{t} \frac{\Delta s}{r(s)}
$$

If

$$
\limsup _{t \rightarrow \infty} \int_{a}^{t} R(\sigma(s)) p(s)\left(\frac{\tau(s)}{\sigma(s)}\right)^{r} \Delta s=\infty,
$$

then (1.1) is oscillatory on $[a, \infty)$. 
Proof. Suppose that (1.1) has a nonoscillatory solution $x(t)$. We may assume without loss of generality that $x(t)>0$ and $x(\tau(t))>0$ for all $t \geq t_{1}>a$. So by Lemma 3.2, (3.3) holds. Define the function $\omega$ by

$$
\omega(t)=\frac{R(t) r(t) x^{\Delta}(t)}{x^{\gamma}(t)}
$$

Then, $\omega(t)>0$ and using (2.5) we get

$$
\begin{aligned}
\omega^{\Delta} & =\left\{\frac{1}{r} r x^{\Delta}+R^{\sigma}\left(r x^{\Delta}\right)^{\Delta}\right\}\left(x^{-\gamma}\right)^{\sigma}+\operatorname{Rr} x^{\Delta}\left(x^{-\gamma}\right)^{\Delta} \\
& =x^{\Delta}\left(x^{-\gamma}\right)^{\sigma}-R^{\sigma} p\left(\frac{x \circ \tau}{x \circ \sigma}\right)^{\gamma}+\operatorname{Rr} x^{\Delta}\left(x^{-\gamma}\right)^{\Delta} .
\end{aligned}
$$

By Lemma 3.1, for each $k \in(0,1)$, there exists a constant $t_{2}=\max \left\{t_{1}, t_{k}\right\}$, such that

$$
\frac{x(\tau(t))}{\tau(t)} \geq k \frac{x(\sigma(t))}{\sigma(t)}
$$

note that $\gamma>1,\left(x^{-\gamma}\right)^{\Delta} \leq 0$, (3.1) and (3.3) imply

$$
\begin{aligned}
\left(x^{1-\gamma}\right)^{\Delta}(t) & =(1-\gamma) \int_{0}^{1}\left[h x^{\sigma}(t)+(1-h) x(t)\right]^{-\gamma} x^{\Delta}(t) \mathrm{d} h \\
& \leq(1-\gamma) \int_{0}^{1}\left[h x^{\sigma}(t)+(1-h) x^{\sigma}(t)\right]^{-\gamma} x^{\Delta}(t) \mathrm{d} h=(1-\gamma)\left(x^{\sigma}(t)\right)^{-\gamma} x^{\Delta}(t) .
\end{aligned}
$$

So,

$$
\omega^{\Delta} \leq \frac{\left(x^{1-\gamma}\right)^{\Delta}}{1-\gamma}-k^{\gamma} R^{\sigma} p\left(\frac{\tau}{\sigma}\right)^{\gamma}
$$

Upon integration, we arrive at

$$
\begin{aligned}
\int_{t_{2}}^{t} R(\sigma(s)) p(s)\left(\frac{\tau(s)}{\sigma(s)}\right)^{\gamma} \Delta s & \leq k^{-\gamma} \int_{t_{2}}^{t}\left(\frac{x^{1-\gamma}}{1-\gamma}-\omega\right)^{\Delta}(s) \Delta s \\
& =k^{-\gamma}\left(\frac{x^{1-\gamma}(t)}{1-\gamma}-\omega(t)-\frac{x^{1-\gamma}\left(t_{2}\right)}{1-\gamma}+\omega\left(t_{2}\right)\right) \\
& \leq k^{-\gamma}\left(\frac{x^{1-\gamma}\left(t_{2}\right)}{\gamma-1}+\omega\left(t_{2}\right)\right)
\end{aligned}
$$

which contradicts (4.3). The proof is complete. 
Remark 3.5. Theorem 3.4 includes results of Akin-Bohner et al. [7, Theorem 4.5].

Putting $r(t) \equiv 1$, that is, $R(t)=t$ in Theorem 3.4, we obtain the following corollary.

Corollary 3.6. Assume $r(t) \equiv 1, \gamma>1$. If

$$
\limsup _{t \rightarrow \infty} \int_{a}^{t} p(s) \frac{(\tau(s))^{\gamma}}{(\sigma(s))^{\gamma-1}} \Delta s=\infty
$$

then (1.1) is oscillatory on $[a, \infty)$.

Theorem 3.7. Assume that (1.8) holds, $r \in C_{\mathrm{rd}}^{1}([a, \infty), \mathbb{R})$, and $r^{\Delta}(t) \geq 0, r \geq 1$. Furthermore, assume that there exists a function $z \in C_{\mathrm{rd}}^{1}([a, \infty), \mathbb{R})$ such that

$$
\limsup _{t \rightarrow \infty} \int_{a}^{t}\left(p(s)\left(\frac{\tau(s)}{\sigma(s)}\right)^{\gamma}(z(\sigma(s)))^{2}-M^{\gamma-1} r(s)\left(z^{\Delta}(s)\right)^{2}\right) \Delta s=\infty
$$

holds for all constants $M>0$. Then (1.1) is oscillatory on $[a, \infty)$.

Proof. Suppose that (1.1) has a nonoscillatory solution $x$. We may assume without loss of generality that $x(t)>0$ and $x(\tau(t))>0$ for all $t \geq t_{1}>a$. So by Lemma 3.2, (3.3) holds. Define the function $\omega(t)$ by

$$
\omega(t)=\frac{z^{2}(t) r(t) x^{\Delta}(t)}{x^{\gamma}(t)}
$$

Then, using (2.5) we get

$$
\omega^{\Delta}=\left(\frac{z^{2}}{x^{\gamma}}\right)^{\sigma}\left(r x^{\Delta}\right)^{\Delta}+r x^{\Delta}\left(\frac{z^{2}}{x^{\gamma}}\right)^{\Delta} .
$$

So, from (1.1) and Lemma 3.3, we have

$$
\omega^{\Delta}=-p\left(z^{\sigma}\right)^{2}\left(\frac{x \circ \tau}{x^{\sigma}}\right)^{\gamma}+\frac{r x^{\Delta}}{\left(x^{\gamma}\right)^{\Delta}}\left(z^{\Delta}\right)^{2}-\frac{r x^{\Delta}}{\left(x^{\gamma}\right)^{\Delta}}\left(x x^{\sigma}\right)^{\gamma}\left(\left(\frac{z}{x^{\gamma}}\right)^{\Delta}\right)^{2}
$$

By Lemma 3.1, for each $k \in(0,1)$, there exists a constant $t_{2}=\max \left\{t_{1}, t_{k}\right\}$, such that

$$
\frac{x(\tau(t))}{\tau(t)} \geq k \frac{x(\sigma(t))}{\sigma(t)}
$$

note that $r \geq 1$, (3.1) and (3.3) imply

$$
\left(x^{\gamma}\right)^{\Delta}(t) \geq \gamma(x(t))^{\gamma-1} x^{\Delta}(t) \geq \gamma\left(x\left(t_{1}\right)\right)^{\gamma-1} x^{\Delta}(t)
$$


then

$$
\frac{1}{k^{\gamma}} \omega^{\Delta} \leq-p\left(z^{\sigma}\right)^{2}\left(\frac{\tau}{\sigma}\right)^{\gamma}+M^{\gamma-1} r\left(z^{\Delta}\right)^{2}
$$

where $M=\left(k^{\gamma /(\gamma-1)} \gamma^{1 /(\gamma-1)} x\left(t_{1}\right)\right)^{-1}$ if $\gamma>1$. If $\gamma=1$, we chose $M=1$.

Therefore,

$$
\int_{t_{1}}^{t}\left(p(s)\left(\frac{\tau(s)}{\sigma(s)}\right)^{\gamma}(z(\sigma(s)))^{2}-M^{\gamma-1} r(s)\left(z^{\Delta}(s)\right)^{2}\right) \Delta s \leq-\frac{1}{k^{\gamma}} \int_{t_{1}}^{t} \omega^{\Delta}(s) \Delta s \leq \frac{1}{k^{\gamma}} \omega\left(t_{1}\right),
$$

which contradicts (3.18). The proof is complete.

Remark 3.8. Theorem 3.7 not only includes results of Agarwal et al. [14, Theorem 4.8], AkinBohner et al. [7, Theorem 4.1], and Han et al. [17, Theorem 3.2], but also improves conditions of Agarwal et al. [14, Theorem 4.8] and Han et al. [17, Theorem 3.2].

From Theorem 3.7, we can obtain different conditions for oscillation of all solutions of (1.1) with different choices of $z(t)$.

For example, let $z=\sqrt{t}, z \equiv 1$. Now Theorem 3.4 yields the following results.

Corollary 3.9. Assume that (1.8) holds, $r \in C_{\mathrm{rd}}^{1}([a, \infty), \mathbb{R})$, and $r^{\Delta}(t) \geq 0, r \geq 1$. If

$$
\limsup _{t \rightarrow \infty} \int_{a}^{t}\left(p(s) \sigma(s)\left(\frac{\tau(s)}{\sigma(s)}\right)^{\gamma}-\frac{M^{\gamma-1} r(s)}{(\sqrt{s}+\sqrt{\sigma(s)})^{2}}\right) \Delta s=\infty
$$

holds for all constants $M>0$, then (1.1) is oscillatory on $[a, \infty)$.

Sometimes the following criterion is easier to check than the one given in Corollary 3.6, but it follows easily from Corollary 3.6 as we always have $\sigma(t) \geq t$ for all $t \in \mathbb{T}$.

Corollary 3.10. Assume that (1.8) holds, $r \in C_{\mathrm{rd}}^{1}([a, \infty), \mathbb{R})$, and $r^{\Delta}(t) \geq 0, r \geq 1$. If

$$
\limsup _{t \rightarrow \infty} \int_{a}^{t}\left(p(s) \sigma(s)\left(\frac{\tau(s)}{\sigma(s)}\right)^{\gamma}-\frac{M^{\gamma-1} r(s)}{4 s}\right) \Delta s=\infty
$$

holds for all constants $M>0$, then (1.1) is oscillatory on $[a, \infty)$.

Now, using Lemma 3.2, we can give some sufficient conditions when (1.9) holds, which guarantee that every solution of (1.1) oscillates or converges to zero in $[a, \infty)$. 
Theorem 3.11. Assume that (1.9) holds, $r \in C_{\mathrm{rd}}^{1}([a, \infty), \mathbb{R})$, and $r^{\Delta}(t) \geq 0$, and $\gamma \geq 1$. Furthermore, assume that there exists a positive function $z \in C_{\mathrm{rd}}^{1}([a, \infty), \mathbb{R})$ such that $(3.18)$ holds. If

$$
\int_{a}^{\infty}\left[\frac{1}{r(t)} \int_{a}^{t} p(s) \Delta s\right] \Delta t=\infty
$$

then every solution of (1.1) is oscillatory or converges to zero on $[a, \infty)$.

Theorem 3.12. Assume that (1.8) holds, $r \in C_{\mathrm{rd}}^{1}([a, \infty), \mathbb{R})$, and $r^{\Delta}(t) \geq 0, r \leq 1$. Furthermore, assume that there exists a function $z \in C_{\mathrm{rd}}^{1}([a, \infty), \mathbb{R})$ such that

$$
\limsup _{t \rightarrow \infty} \int_{a}^{t}\left(p(s)\left(\frac{\tau(s)}{\sigma(s)}\right)^{\gamma}(z(\sigma(s)))^{2}-K^{\gamma-1}(\sigma(s))^{1-\gamma} r(s)\left(z^{\Delta}(s)\right)^{2}\right) \Delta s=\infty
$$

holds for all constants $K>0$. Then (1.1) is oscillatory on $[a, \infty)$.

Proof. We assume that (1.1) has a nonoscillatory solution such that $x(t)>0$, and $x(\tau(t))>0$, for all $t \geq t_{1}>a$. By Lemma 3.2, we obtain (3.3). We calculate

$$
x(t)=x\left(t_{1}\right)+\int_{t_{1}}^{t} x^{\Delta}(s) \Delta s \leq \alpha+\beta t
$$

where $\alpha=x\left(t_{1}\right)-t_{1} x^{\Delta}\left(t_{1}\right)$ and $\beta=x^{\Delta}\left(t_{1}\right)$. By putting $L=|\alpha|+\beta$ and $t_{2} \geq \max \left\{t_{1}, 1\right\}$, we find that

$$
x(t) \leq L t \quad \forall t \geq t_{2} .
$$

Now note that $\gamma \leq 1,(3.1)$ and (3.31) imply

$$
\begin{aligned}
\left((x(t))^{\gamma}\right)^{\Delta} & =\gamma \int_{0}^{1}\left[h x^{\sigma}(t)+(1-h) x(t)\right]^{\gamma-1} x^{\Delta}(t) \mathrm{d} h \\
& \geq \gamma \int_{0}^{1}\left[h x^{\sigma}(t)+(1-h) x^{\sigma}(t)\right]^{\gamma-1} x^{\Delta}(t) \mathrm{d} h=\gamma\left(x^{\sigma}(t)\right)^{\gamma-1} x^{\Delta}(t) .
\end{aligned}
$$

So,

$$
\left((x(t))^{\gamma}\right)^{\Delta} \geq \gamma(L \sigma(t))^{\gamma-1} x^{\Delta}(t)=\frac{(\sigma(t))^{\gamma-1}}{K_{1}^{\gamma-1}} x^{\Delta}(t),
$$


where we put $K_{1}=\left(\gamma^{1 /(\gamma-1)} L\right)^{-1}$ if $\gamma<1$. If $\gamma=1$, we chose $K_{1}=1$. Note $K_{1}>0$. Now define the function $\omega$ by (3.19). Therefore, using (3.21), Lemma 3.1, for each $k \in(0,1)$, there exists a constant $t_{2}=\max \left\{t_{1}, t_{k}\right\}$, such that

$$
\frac{x(\tau(t))}{\tau(t)} \geq k \frac{x(\sigma(t))}{\sigma(t)}
$$

noting that $\gamma \leq 1,(3.3)$ and (3.33), we obtain

$$
\frac{1}{k r} \omega^{\Delta} \leq-p\left(z^{\sigma}\right)^{2}\left(\frac{\tau}{\sigma}\right)^{\gamma}+K^{\gamma-1}(\sigma)^{1-\gamma} r\left(z^{\Delta}\right)^{2}
$$

where $K=\left(k^{\gamma /(\gamma-1)} \gamma^{1 /(\gamma-1)} L\right)^{-1}$ if $\gamma<1$. If $\gamma=1$, we chose $K=1$. Upon integration, we arrive at

$$
\int_{t_{2}}^{t}\left(p(s)\left(\frac{\tau(s)}{\sigma(s)}\right)^{\gamma}(z(\sigma(s)))^{2}-K^{\gamma-1}(\sigma(s))^{1-\gamma} r(s)\left(z^{\Delta}(s)\right)^{2}\right) \Delta s \leq-\frac{1}{k^{\gamma}} \int_{t_{2}}^{t} \omega^{\Delta}(s) \Delta s \leq \frac{1}{k^{r}} \omega\left(t_{2}\right),
$$

which contradicts (3.29). The proof is complete.

Remark 3.13. Theorem 3.12 includes results of Akin-Bohner et al. [7, Theorem 5.1] and Han et al. [17, Theorem 3.4] and improves conditions of Han [17, Theorem 3.4].

From Theorem 3.12, we can obtain different conditions for oscillation of all solutions of (1.1) with different choices of $z(t)$.

For example, let $z=\sqrt{t}, z \equiv 1$. Now Theorem 3.12 yields the following results.

Corollary 3.14. Assume that (1.8) holds, $r \in C_{\mathrm{rd}}^{1}([a, \infty), \mathbb{R})$, and $r^{\Delta}(t) \geq 0, r \leq 1$. If

$$
\limsup _{t \rightarrow \infty} \int_{a}^{t}\left(p(s) \sigma(s)\left(\frac{\tau(s)}{\sigma(s)}\right)^{\gamma}-\frac{K^{\gamma-1}(\sigma(s))^{1-\gamma} r(s)}{(\sqrt{s}+\sqrt{\sigma(s)})^{2}}\right) \Delta s=\infty
$$

holds for all constants $K>0$, then (1.1) is oscillatory on $[a, \infty)$.

Sometimes the following criterion is easier to check than the one given in Corollary 3.14, but it follows easily from Corollary 3.14 as we always have $\sigma(t) \geq t$ for all $t \in \mathbb{T}$.

Corollary 3.15. Assume that (1.8) holds, $r \in C_{\mathrm{rd}}^{1}([a, \infty), \mathbb{R})$, and $r^{\Delta}(t) \geq 0, r \leq 1$. If

$$
\limsup _{t \rightarrow \infty} \int_{a}^{t}\left(p(s) \sigma(s)\left(\frac{\tau(s)}{\sigma(s)}\right)^{\gamma}-(\sigma(s))^{1-\gamma} r(s) \frac{K^{\gamma-1}}{4 s}\right) \Delta s=\infty
$$

holds for all constants $K>0$, then (1.1) is oscillatory on $[a, \infty)$. 


\section{Applications}

In this section, we give one example to illustrate our main results. To obtain the conditions for oscillation, we will use the following fact:

$$
\int_{a}^{\infty} \frac{\Delta t}{t^{p}}=\infty \text { if } 0 \leq p \leq 1
$$

For more details, we refer the reader to [4, Theorem 5.68].

Akin-Bohner et al. [7] considered the second-order dynamic equations on time scales

$$
\left(r x^{\Delta}\right)^{\Delta}(t)+p(t) x^{\gamma}(\sigma(t))=0, \quad t \in \mathbb{T},
$$

where $r>1$ is a quotient of odd positive integer, $r$ and $p$ are positive, real-valued rdcontinuous functions defined on $\mathbb{T}$, and established some new oscillation criteria of (4.2).

Theorem 4.1 (Akin-Bohner et al. [7, Theorem 4.5]). Assume that (1.8) holds. If

$$
\limsup _{t \rightarrow \infty} \int_{a}^{t} R(\sigma(s)) p(s) \Delta s=\infty
$$

then (4.2) is oscillatory on $[a, \infty)$.

We note that (1.1) becomes (4.2) when $\tau(t) \equiv \sigma(t)$, and Theorem 3.4 becomes Theorem 4.1, so Theorem 3.4 essentially includes results of Akin-Bohner et al. [7, Theorem 4.5].

Example 4.2. Consider the second-order delay dynamic equations on time scales

$$
\left(t^{1 / 3} x^{\Delta}(t)\right)^{\Delta}+\frac{1}{t^{1 / 3}} \frac{(\sigma(t))^{\gamma-1}}{(\tau(t))^{\gamma}}(x(\tau(t)))^{\gamma}=0, \quad t \in[1, \infty),
$$

where $r(t)=t^{1 / 3}, p(t)=1 / t^{1 / 3}(\sigma(t))^{\gamma-1} /(\tau(t))^{\gamma}, \gamma>1$. By Corollary 3.10, we have

$$
\begin{gathered}
\int_{1}^{\infty} \frac{1}{r(t)} \Delta t=\infty, \\
\limsup _{t \rightarrow \infty} \int_{1}^{t}\left(p(s) \frac{(\tau(s))^{\gamma}}{(\sigma(s))^{\gamma-1}}-\frac{r(s)}{4 s} M^{\gamma-1}\right) \Delta s=\limsup _{t \rightarrow \infty} \int_{1}^{t} \frac{1}{s^{2 / 3}}\left(s^{1 / 3}-\frac{M^{\gamma-1}}{4}\right) \Delta s .
\end{gathered}
$$


Let $\left(s^{1 / 3}-M^{\gamma-1} / 4\right)>1$, then $s>\left(1+M^{\gamma-1} / 4\right)^{3}$, pick $t_{1}=\left(1+M^{\gamma-1} / 4\right)^{3}$, so $\left(s^{1 / 3}-M^{\gamma-1} / 4\right)>1$ for all $s>t_{1}$. Therefore,

$$
\begin{aligned}
\limsup _{t \rightarrow \infty} \int_{1}^{t} \frac{1}{s^{2 / 3}}\left(s^{1 / 3}-\frac{M^{\gamma-1}}{4}\right) \Delta s= & \int_{1}^{t_{1}} \frac{1}{s^{2 / 3}}\left(s^{1 / 3}-\frac{M^{\gamma-1}}{4}\right) \Delta s \\
& +\limsup _{t \rightarrow \infty} \int_{t_{1}}^{t} \frac{1}{s^{2 / 3}}\left(s^{1 / 3}-\frac{M^{\gamma-1}}{4}\right) \Delta s \\
\geq & \int_{1}^{t_{1}} \frac{1}{s^{2 / 3}}\left(s^{1 / 3}-\frac{M^{\gamma-1}}{4}\right) \Delta s+\limsup _{t \rightarrow \infty} \int_{t_{1}}^{t} \frac{1}{s^{2 / 3}} \Delta s=\infty
\end{aligned}
$$

for all constants $M>0$. Then (4.4) is oscillatory on $[1, \infty)$.

\section{Acknowledgments}

This research is supported by the Natural Science Foundation of China $(60774004,60904024)$, China Postdoctoral Science Foundation funded project (20080441126, 200902564), Shandong Postdoctoral funded project (200802018), and supported by the Natural Science Foundation of Shandong (Y2008A28, ZR2009AL003), also supported by the Fund of Doctoral Program Research of University of Jinan (B0621, XBS0843).

\section{References}

[1] S. Hilger, "Analysis on measure chains—a unified approach to continuous and discrete calculus," Results in Mathematics, vol. 18, no. 1-2, pp. 18-56, 1990.

[2] R. P. Agarwal, M. Bohner, D. O'Regan, and A. Peterson, "Dynamic equations on time scales: a survey," Journal of Computational and Applied Mathematics, vol. 141, no. 1-2, pp. 1-26, 2002.

[3] M. Bohner and A. Peterson, Dynamic Equations on Time Scales. An Introduction with Applications, Birkhäuser, Boston, Mass, USA, 2001.

[4] M. Bohner and A. Peterson, Eds., Advances in Dynamic Equations on Time Scales, Birkhäuser, Boston, Mass, USA, 2003.

[5] E. Akin-Bohner and J. Hoffacker, "Oscillation properties of an Emden-Fowler type equation on discrete time scales," Journal of Difference Equations and Applications, vol. 9, no. 6, pp. 603-612, 2003.

[6] E. Akin-Bohner and J. Hoffacker, "Solution properties on discrete time scales," Journal of Difference Equations and Applications, vol. 9, no. 1, pp. 63-75, 2003.

[7] E. Akin-Bohner, M. Bohner, and S. H. Saker, "Oscillation criteria for a certain class of second order Emden-Fowler dynamic equations," Electronic Transactions on Numerical Analysis, vol. 27, pp. 1-12, 2007.

[8] M. Bohner and S. H. Saker, "Oscillation of second order nonlinear dynamic equations on time scales," The Rocky Mountain Journal of Mathematics, vol. 34, no. 4, pp. 1239-1254, 2004.

[9] L. Erbe, "Oscillation results for second-order linear equations on a time scale," Journal of Difference Equations and Applications, vol. 8, no. 11, pp. 1061-1071, 2002.

[10] L. Erbe, A. Peterson, and S. H. Saker, "Oscillation criteria for second-order nonlinear dynamic equations on time scales," Journal of the London Mathematical Society, vol. 67, no. 3, pp. 701-714, 2003.

[11] T. Li, Z. Han, S. Sun, and C. Zhang, "Forced oscillation of second-order nonlinear dynamic equations on time scales," Electronic Journal of Qualitative Theory of Differential Equations, vol. 60, pp. 1-8, 2009.

[12] S. H. Saker, "Oscillation criteria of second-order half-linear dynamic equations on time scales," Journal of Computational and Applied Mathematics, vol. 177, no. 2, pp. 375-387, 2005. 
[13] S. H. Saker, "Oscillation of nonlinear dynamic equations on time scales," Applied Mathematics and Computation, vol. 148, no. 1, pp. 81-91, 2004.

[14] R. P. Agarwal, M. Bohner, and S. H. Saker, "Oscillation of second order delay dynamic equations," The Canadian Applied Mathematics Quarterly, vol. 13, no. 1, pp. 1-17, 2005.

[15] R. P. Agarwal, D. O’Regan, and S. H. Saker, "Oscillation criteria for second-order nonlinear neutral delay dynamic equations," Journal of Mathematical Analysis and Applications, vol. 300, no. 1, pp. 203217, 2004.

[16] M. Bohner, "Some oscillation criteria for first order delay dynamic equations," Far East Journal of Applied Mathematics, vol. 18, no. 3, pp. 289-304, 2005.

[17] Z. Han, S. Sun, and B. Shi, "Oscillation criteria for a class of second-order Emden-Fowler delay dynamic equations on time scales," Journal of Mathematical Analysis and Applications, vol. 334, no. 2, pp. 847-858, 2007.

[18] Z. Han, B. Shi, and S. Sun, "Oscillation criteria for second-order delay dynamic equations on time scales," Advances in Difference Equations, vol. 2007, Article ID 70730, 16 pages, 2007.

[19] Z. L. Han, B. Shi, and S. R. Sun, "Oscillation of second-order delay dynamic equations on time scales," Acta Scientiarum Naturalium Universitatis Sunyatseni, vol. 46, no. 6, pp. 10-13, 2007.

[20] Z. Han, T. Li, S. Sun, and C. Zhang, "Oscillation for second-order nonlinear delay dynamic equations on time scales," Advances in Difference Equations, vol. 2009, Article ID 756171, 13 pages, 2009.

[21] Z. Han, T. Li, S. Sun, and C. Zhang, "Oscillation behavior of third order neutral Emden-Fowler delay dynamic equations on time scales," Advances in Difference Equations, vol. 2010, Article ID 586312, 23 pages, 2010.

[22] T. Li, Z. Han, S. Sun, and D. Yang, "Existence of nonoscillatory solutions to second-order neutral delay dynamic equations on time scales," Advances in Difference Equations, vol. 2009, Article ID 562329, 10 pages, 2009.

[23] Y. Şahiner, "Oscillation of second-order delay differential equations on time scales," Nonlinear Analysis: Theory, Methods \& Applications, vol. 63, no. 5-7, pp. e1073-e1080, 2005.

[24] S. H. Saker, "Oscillation of second-order nonlinear neutral delay dynamic equations on time scales," Journal of Computational and Applied Mathematics, vol. 187, no. 2, pp. 123-141, 2006.

[25] S.-R. Sun, Z.-L. Han, and C.-H. Zhang, "Oscillation criteria of second-order Emden-Fowler neutral delay dynamic equations on time scales," Journal of Shanghai Jiaotong University, vol. 42, no. 12, pp. 2070-2075, 2008.

[26] S. Sun, Z. Han, and C. Zhang, "Oscillation of second-order delay dynamic equations on time scales," Journal of Applied Mathematics and Computing, vol. 30, no. 1-2, pp. 459-468, 2009.

[27] Y. Sun, Z. Han, T. Li, and G. Zhang, "Oscillation criteria for second-order quasilinear neutral delay dynamic equations on time scales," Advances in Difference Equations, vol. 2010, Article ID 512437, 14 pages, 2010.

[28] L. Erbe, A. Peterson, and S. H. Saker, "Oscillation criteria for second-order nonlinear delay dynamic equations," Journal of Mathematical Analysis and Applications, vol. 333, no. 1, pp. 505-522, 2007.

[29] B. G. Zhang and Z. Shanliang, "Oscillation of second-order nonlinear delay dynamic equations on time scales," Computers \& Mathematics with Applications, vol. 49, no. 4, pp. 599-609, 2005.

[30] L. Erbe, "Oscillation criteria for second order nonlinear delay equations," Canadian Mathematical Bulletin, vol. 67, no. 16, pp. 49-56, 1973.

[31] R. P. Agarwal, S.-L. Shieh, and C.-C. Yeh, "Oscillation criteria for second-order retarded differential equations," Mathematical and Computer Modelling, vol. 26, no. 4, pp. 1-11, 1997.

[32] S. Z. Chen and L. H. Erbe, "Riccati techniques and discrete oscillations," Journal of Mathematical Analysis and Applications, vol. 142, no. 2, pp. 468-487, 1989.

[33] S. Z. Chen and L. H. Erbe, "Oscillation and nonoscillation for systems of selfadjoint second-order difference equations," SIAM Journal on Mathematical Analysis, vol. 20, no. 4, pp. 939-949, 1989.

[34] J. Ohriska, "Oscillation of second order delay and ordinary differential equation," Czechoslovak Mathematical Journal, vol. 34(109), no. 1, pp. 107-112, 1984.

[35] E. Thandapani, K. Ravi, and J. R. Graef, "Oscillation and comparison theorems for half-linear secondorder difference equations," Computers \& Mathematics with Applications, vol. 42, no. 6-7, pp. 953-960, 2001.

[36] Z. Zhang, J. Chen, and C. Zhang, "Oscillation of solutions for second-order nonlinear difference equations with nonlinear neutral term," Computers \& Mathematics with Applications, vol. 41, no. 12, pp. 1487-1494, 2001. 